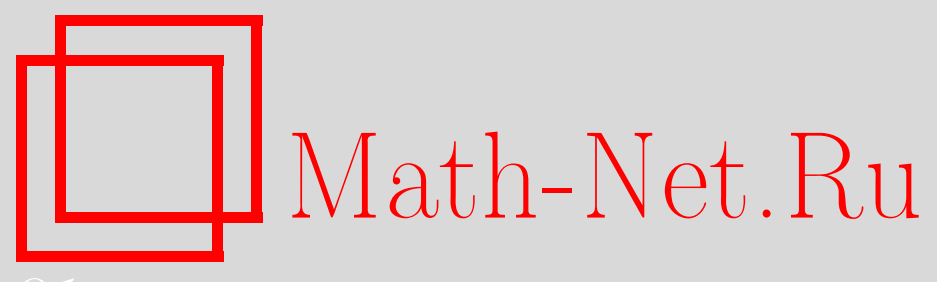

А. Б. Еловиков, Однопорожденные композиционные фор-
мации, Дискрет. матем., 2001, том 13, выпуск 3, 153-160
DOI: https://doi.org $/ 10.4213 /$ dm293

Использование Общероссийского математического портала Math-Net.Ru подразумевает, что вы прочитали и согласны с пользовательским соглашением http://www.mathnet.ru/rus/agreement

Параметры загрузки:

IP: 52.87 .193 .239

26 апреля 2023 г., 13:23:59 
УдК 512.542

\title{
Однопорожденные композиционные формации
}

\author{
(c) 2001 г. А. Б. Еловиков
}

\begin{abstract}
В работе описывается класс факторизуемых однопорожденных композиционных формаций через установление необходимых и достаточных условий факторизации. Рассматриваются только конечные группы.
\end{abstract}

Однопорожденные формации конечных групп были введены в рассмотрение основателем теории формаций В. Гашюцем. Исследованию факторизуемых однопорожденных локальных формаций посвящен ряд работ (см., например, [1-3]). А. Н. Скиба в книге [4] описал все возможные факторизации однопорожденных локальных формаций, там же был поставлен вопрос: можно ли описать все возможные несократимые факторизации однопорожденных композиционных формаций? (см. [4], вопрос 3.5.21).

В настоящей работе описывается обширный класс факторизуемых однопорожденных композиционных формаций через установление необходимых и достаточных условий факторизации. Основные результаты статьи анонсированы в [10]. Рассматриваются только конечные группы. Все необходимые определения и обозначения можно найти в [4-6]. Приведем только некоторые из них. Через $G=[A] B$ обозначают полупрямое произведение групп $A$ и $B$, где $A$ нормальна в $G ; C F(f)$ - композиционная формация, определяемая композиционным спутником $f$. С целью компактного изложения материала композиционные формации ( $n$-кратно композиционные формации) коротко будем называть $c$-формациями $\left(c_{n}\right.$-формациями), а композиционные спутники ( $n$-кратно композиционные спутники) $c$-спутниками $\left(c_{n}\right.$-спутниками). Через $\mathfrak{G}, \mathfrak{I}, \mathfrak{S}$ обозначают соответственно класс всех конечных групп, класс всех конечных простых групп, класс всех конечных разрешимых групп.

Перейдем к изложению полученных результатов.

Лемма 1. Пусть $A \in \mathfrak{G}$, тогда в формации $\mathrm{cform} A$ содержитсл лишъ конечное множество наследственных с-формаций.

Доказательство. Обозначим $\theta$ полную решетку всех наследственных формаций. Пусть $\mathfrak{M}=\theta^{c}$ form $A$ - пересечение всех $c$-формаций, содержащих $A$ и имеющих хотя бы один $\theta$-значный спутник. Очевидно, что $\operatorname{cform} A \subseteq \mathfrak{M}$. Покажем, что в $\mathfrak{M}$ содержится лишь конечное число наследственных $c$-формаций.

Пусть $\mathfrak{F}-$ произвольная наследственная $c$-формация такая, что $\mathfrak{F} \subseteq \mathfrak{M}$. По следствию 1.2 из [7] $\mathfrak{F} \in \theta^{c}$. Пусть $f$ и $m$ - минимальные наследственные $c$-спутники 
формаций $\mathfrak{F}$ и $\mathfrak{M}$, соответственно, тогда по следствию 2.1 из [7] $f \leqslant m$. По лемме 6 из [8]

$$
m(B)=\theta \text { form }\left(A / F_{B}(A)\right),
$$

где $B \in K(A)$. Следовательно, по лемме 8.8 из [5] существует лишь конечное множество наследственных $c$-спутников $t$, меньших $m$. Значит, в $\mathfrak{M}$, а тем более в cform $A$, существует лишь конечное множество наследственных $c$-подформаций.

Лемма доказана.

Лемма 2. Если $\mathfrak{F}=\mathfrak{M H}$, где $\mathfrak{F} u \mathfrak{M}-$ неединичные с-формачии, а $\mathfrak{H}-$ такая формачия, что

$$
K(\mathfrak{H}) \cap \mathfrak{A} \nsubseteq K(\mathfrak{M}) \cap \mathfrak{A} \neq \varnothing,
$$

то множество наследственных с-подформаций формачии F бесконечно.

Доказательство. Пусть $Z_{p} \in K(\mathfrak{H}) \backslash K(\mathfrak{M})$. Ясно, что $\mathfrak{H} \subseteq \mathfrak{F}$. Значит $Z_{p} \in K(\mathfrak{F})$. Формация $\mathfrak{F}$ композиционна. Следовательно, $\mathfrak{N}_{p} \subseteq \mathfrak{F}$. Легко показать, что $\mathfrak{N}_{p} \subseteq \mathfrak{H}$. Пусть $Z_{q} \in K(\mathfrak{M})$. Тогда $q \neq p$ и $\mathfrak{N}_{q} \subseteq \mathfrak{M}$. Для всякого натурального числа $n$ зафиксируем некоторую циклическую группу $P_{n}$ порядка $p^{n}$. Пусть $Q_{n}=Z_{q}$ २ $P_{n}-$ регулярное сплетение. Обозначим через $\mathfrak{F}_{n}$ композиционную формацию, порожденную группой $Q_{n}$. Группа $Q_{n}$ является расширением $q$-группы с помощью $p$-группы. Следовательно,

$$
Q_{n} \in \mathfrak{N}_{q} \mathfrak{N}_{p} \subseteq \mathfrak{M H}=\mathfrak{F} .
$$

Поэтому $\mathfrak{F}_{n} \subseteq \mathfrak{F}$. Поскольку группа $Q_{n}$ метанильпотентна, то $\mathfrak{F}_{n} \subseteq \mathfrak{N}^{2}$. Хорошо известно, что каждая разрешимая $c$-формация является локальной формацией. Значит, ввиду леммы 8.10 из [5] все $c$-подформации формации $\mathfrak{N}^{2}$ наследственны. Тогда $\mathfrak{F}_{n}-$ наследственная $c$-формация в $\mathfrak{F}$.

При доказательстве леммы 8.13 в [5] было показано, что если $n$ и $m-$ различные натуральные числа, то $\mathfrak{F}_{n} \neq \mathfrak{F}_{m}$. Итак, в $\mathfrak{F}$ содержится бесконечное множество наследственных $c$-подформаций $\mathfrak{F}_{1}, \mathfrak{F}_{2}, \ldots$

Лемма доказана.

Используя методы, разработанные в [5] (теорема 7.10) и [8] (теорема 1), несложно показать, что справедливо следующее утверждение.

Лемма 3. Пусть $\mathfrak{M}=C F(m)$, причем $\mathfrak{N}_{\pi(\mathfrak{N})} \subseteq \mathfrak{M}, \mathfrak{H}-$ такая непустал формачия, что $\mathfrak{N}_{q} \mathfrak{H}=\mathfrak{H}$ для любого простого числа $q$ такого, что $Z_{q} \in(K(\mathfrak{H}) \backslash K(\mathfrak{M}))$ (это условие, в частности, выполняется, если $K(\mathfrak{H}) \cap \mathfrak{A} \subseteq K(\mathfrak{M}) \cap \mathfrak{A})$. Тогда формачия $\mathfrak{F}=\mathfrak{M H}$ имеет такой с-спутник $f$, ито $f(A)=m(A) \mathfrak{H}$ для всех $A \in K(\mathfrak{M})$, $f(A)=\mathfrak{H}$, если $A \in K(\mathfrak{H}) \backslash K(\mathfrak{M})$ u $f(A)=\varnothing$ для любого $A \in \mathfrak{I} \backslash K(\mathfrak{M} \cup \mathfrak{H})$.

Замечание 1. Лемма 3 дополняет теоремы 7.9 и 7.10 из [5].

Лемма 4. Пусть $A \in \mathfrak{G} u \mathfrak{F}$ - формация, содержащая лишь конечное число попарно неизоморфных формачионно критических групп. Тогда если $A^{\mathfrak{F}}$ не имеет фраттиниевых $A$-главных факторов, то в формации $\mathfrak{M}=c_{n}$ form $A$ имеется лишь конечное число п-кратно композичионных подформаций.

Доказательство. Проведем индукцию по $n$. Пусть $n=0$. Тогда $\mathfrak{M}=$ form $A$. По теореме 3.44 из [5] $\mathfrak{M}$ имеет лишь конечное множество подформаций. 
Пусть теперь $n>0$ и лемма верна для $n-1$. Обозначим через $m$ минимальный $c_{(n-1)}$-спутник формации $\mathfrak{M}$. По лемме 6 из [8]

$$
m(B)=c_{(n-1)} \text { form }\left(A / F_{B}(A)\right)
$$

для любого $B \in K(A)$ и $m(B)=\varnothing$ для всех $B \in \mathfrak{I} \backslash K(A)$. Ввиду теоремы 3.36 из [5] F-корадикал группы $A / F_{B}(A)$ не содержит фраттиниевых $\left(A / F_{B}(A)\right)$-главных факторов. Значит, по индукции в формации $c_{(n-1)}$ form $\left(A / F_{B}(A)\right)$ имеется лишь конечное множество $(n-1)$-кратно композиционных подформаций. Применяя теперь следствие 5 из [8], заключаем, что в формации $\mathfrak{M}$ содержится лишь конечное множество $n$-кратно композиционных подформаций. Лемма доказана.

Из теоремы 3.49 в [5] и леммы 4 вытекает следующее утверждение.

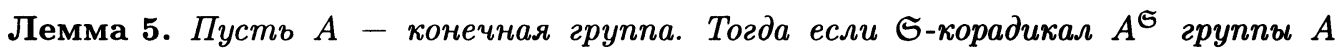
не имеет фраттиниевых $A$-главных факторов, то в формации $c_{n}$ form $A$ имеется лишь конечное множество $c_{n}$-подформаций.

Лемма 6. Пусть $\mathfrak{F} u \mathfrak{H}-\oint о р м а ц и и ~ u \mathfrak{F} \nsubseteq \mathfrak{H}$, причем по крайней мере одна из формачий $\mathfrak{F}, \mathfrak{H}$ разрешима. Если $\mathfrak{F}$ является $c_{n}$-формачией, то в ней содержится минимальная п-кратно композиционная не $\mathfrak{H}$-формачия.

Доказательство. Утверждение очевидно при $\mathfrak{H}=\varnothing$. Пусть $\mathfrak{H} \neq \varnothing$ и $A$ - группа минимального порядка из $\mathfrak{F} \backslash \mathfrak{H}$. Тогда $A$ - монолитическая группа с монолитом $R=A^{\mathfrak{H}}$. Индукцией по $n$ покажем, что в $\mathfrak{M}=c_{n}$ form $A$ имеется лишь конечное множество $c_{n}$-подформаций.

Пусть $n=0$. Тогда $\mathfrak{M}=$ form $A$. По лемме 19.6 из [5] $\mathfrak{M}$ имеет лишь конечное множество подформаций.

Пусть $n>0$ и лемма верна для $n-1$. Обозначим через $m$ минимальный $c_{(n-1)}$ спутник формации $\mathfrak{M}$. По лемме 6 из [8] $m(B)=c_{(n-1)}$ form $\left(A / F_{B}(A)\right)$ для любого $B \in K(A)$ и $m(B)=\varnothing$ для любого $B \in \mathfrak{I} \backslash K(A)$. Если $F_{B}(A) \neq 1$, то, ввиду условия леммы, $A / F_{B}(A) \in \mathfrak{S}$, то есть $\left(A / F_{B}(A)\right)^{\mathfrak{S}}=1$. Тогда по лемме 5 в $m(B)$ имеется лишь конечное множество $c_{(n-1)}$-подформаций. Если же $F_{B}(A)=1$, то $m(B)=c_{(n-1)}$ form $A$ и по индукции число $c_{(n-1)}$-подформаций в $m(B)$ конечно. Следовательно, в формации $\mathfrak{M}$ содержится лишь конечное множество $c_{n}$-подформаций. Так как при этом $\mathfrak{M} \nsubseteq \mathfrak{H}$, но $(1) \subseteq \mathfrak{H}$, то в $\mathfrak{M}$ можно выбрать такую $c_{n}$ - подформацию $\mathfrak{K}$, что $\mathfrak{K} \nsubseteq \mathfrak{H}$, но $\mathfrak{K}_{1} \subseteq \mathfrak{H}$ для каждой собственной $c_{n}$-подформации $\mathfrak{K}_{1}$ из $\mathfrak{K}$.

Лемма доказана.

Лемма 7. Пусть $\mathfrak{F}$ - минимальная композиционная не $\mathfrak{N}^{n}$-формация. Тогда $\mathfrak{F}=\operatorname{cform} G$, где $G-$ такая монолитическая группа с монолитом $P=G^{\mathfrak{N}^{n}}$, ито выполняется одно из следующих условий:

(1) $G=P-$ простая неабелева группа,

(2) $P$ - собственная неабелева подгруппа группъ $G$,

(3) $G=[P] H$, где $P=C_{G}(P)-$-әруппа и $H=[Q] N$, причем

$$
C_{H}(Q)=Q=H^{\mathfrak{N}^{n-1}}
$$


Доказательство. По теореме 7.9 в [5] и теореме 3.2 в [6] формация $\mathfrak{N}^{n}$ имеет такой максимальный внутренний композиционный спутник $h$, что $h\left(Z_{p}\right)=\mathfrak{N}_{p} \mathfrak{N}^{n-1}$ для всех $Z_{p} \in \mathfrak{A}$ и $h(A)=\mathfrak{N}^{n}$ для любой простой неабелевой группы $A$. Ввиду теоремы 1 из [11], $\mathfrak{F}=\operatorname{cform} G$, где $G$ - такая монолитическая группа с монолитом $P=G^{\mathfrak{N}^{n}}$, что выполняется одно из следующих условий:

(1) $G=P-$ простая группа,

(2) $P$ - собственная неабелева подгруппа группы $G$ и $P=G^{h(A)}$ для $A \in K(P)$,

(3) $G=[P] H$, где $P=C_{G}(P)-p$-группа, а $H \neq 1-$ монолитическая группа с монолитом $Q=H^{h(A)}$ для $A \in K(P)$.

Пусть верно условие 1 . Если $G=P=Z_{p}$ для некоторого $p \in \mathbf{P}$, то form $G \subseteq \mathfrak{N} \subseteq$ $\mathfrak{N}^{n}$. Получаем противоречие. Отсюда, $G=P-$ простая неабелева группа.

Пусть верно условие 3 . Поскольку $H \notin h\left(Z_{p}\right)=\mathfrak{N}_{p} \mathfrak{N}^{n-1}$ для $Z_{p} \in K(P)$, то $H \notin \mathfrak{N}$. Из следствия 1 из [11] заключаем, что $\Phi(H)=1$, тогда $H=[Q] N$. Так как

$$
G / P \cong H \in \mathfrak{N}^{n} \subseteq \mathfrak{S}
$$

то $Q$ абелева. Из монолитичности $H$ следует, что $Q=C_{H}(Q)$.

Поскольку $Q$ - абелева группа, $Q \subseteq F(H)$. Но $F(H) \subseteq C_{H}(Q)=Q$. Следовательно, $Q=F(H)$. Так как $H \in \mathfrak{N}^{n}$, то $H^{\mathfrak{N}^{n-1}} \in \mathfrak{N}$, а значит, $H^{\mathfrak{N}^{n-1}} \subseteq F(H)=Q$. Из монолитичности $H$ получим, что $Q=H^{\mathfrak{N}^{n-1}}$.

Лемма доказана.

Теорема 1. Если произведение $\mathfrak{M H}$ неединичной с-формачии $\mathfrak{M}$ такой, что $\mathfrak{N}_{\pi(\mathfrak{N})} \subseteq \mathfrak{M}$, и неединичной формачии $\mathfrak{H} \neq \varnothing$ является однопорожденной $c$-формацией, то выполняются следующие условия:

(1) $K(\mathfrak{H}) \cap \mathfrak{A} \subseteq K(\mathfrak{M}) \cap \mathfrak{A}$,

(2) $\mathfrak{M}$ - метанильпотентная однопорожденная с-формация,

(3) $\pi(\mathfrak{H}) \cap \pi(m(B))=\varnothing$ для всех $B \in K(\mathfrak{M})$, где $m-$ минималъный $c$-спутник формации $\mathfrak{M}$,

(4) если $|\pi(\mathfrak{M})|>1$, то $\mathfrak{H}$ - однопорожденная формация, причем $\mathfrak{M} \nsubseteq \mathfrak{N}$ влечет включение $\mathfrak{H} \subseteq \mathfrak{A}$.

Доказательство. Пусть $\mathfrak{F}=\mathfrak{M H}$ и $\mathfrak{F}=\operatorname{cform} A$. Допустим, что

$$
K(\mathfrak{H}) \cap \mathfrak{A} \nsubseteq K(\mathfrak{M}) \cap \mathfrak{A} .
$$

Так как по условию $\mathfrak{N}_{\pi(\mathfrak{M})} \subseteq \mathfrak{M}$, то $K(\mathfrak{M}) \cap \mathfrak{A} \neq \varnothing$. Тогда по лемме 2 в формации $\mathfrak{F}$ содержится бесконечное множество наследственных $c$-подформаций, что противоречит лемме 1 . Значит,

$$
K(\mathfrak{H}) \cap \mathfrak{A} \subseteq K(\mathfrak{M}) \cap \mathfrak{A}
$$

то есть формации $\mathfrak{M}$ и $\mathfrak{H}$ удовлетворяют условию 1.

Пусть $h-$ минимальный $c$-спутник формации $\mathfrak{F}$. По лемме 3 формация $\mathfrak{F}$ имеет также $c$-спутник $f$ такой, что $f(B)=m(B) \mathfrak{H}$ для всех $B \in K(\mathfrak{M})$, где $m-$ минимальный с-спутник формации $\mathfrak{M}, f(B)=\mathfrak{H}$ при всяком $B \in K(\mathfrak{H}) \backslash K(\mathfrak{M})$ и $f(B)=\varnothing$, 
если $A \in \mathfrak{I} \backslash K(\mathfrak{M} \cup \mathfrak{H})$. Поскольку $m$ - внутренний спутник формации $\mathfrak{M}$, то $f$ - внутренний спутник формации $\mathfrak{F}$. Предположим, что найдутся такие различные простые числа $p$ и $q$, что в формации $m\left(Z_{p}\right)$ содержится группа $Z_{q}$ и $q \in \pi(\mathfrak{H})$. Пусть $H-$ некоторая $q d$-группа из $\mathfrak{H}$. Для всякого натурального числа $n$ через $H_{n}$ обозначим регулярное сплетение

$$
Z_{q} \prec H^{n}=[K] H^{n},
$$

где $K$ - база сплетения $H_{n}$, а $H^{n}$ обозначает некоторую группу, являющуюся прямым произведением $n$ изоморфных копий группы $H$. Так как

$$
H_{n} / K \cong H^{n} \in \mathfrak{H}
$$

то есть $\left(H_{n}\right)^{\mathfrak{H}} \subseteq K \in m\left(Z_{p}\right)$, то $H_{n} \in m\left(Z_{p}\right) \mathfrak{H}$. По лемме 18.8(b) из [12] $O_{p}\left(H_{n}\right)=1$. Следовательно, по теореме работы [9] $H_{n} \in h\left(Z_{p}\right)$. Если $L-$ подгруппа порядка $q$ из $H$, то по лемме $18.8(\mathrm{a})$ из [12] группа $T_{n}=Z_{q} 2 L^{n}$ изоморфно вкладывается в $H_{n}$. Тогда

$$
T_{n} \in \operatorname{sform}\left(A / F_{Z_{p}}(A)\right)
$$

для всех $n \in \mathrm{N}$. Ввиду леммы 3.1 .7 из [4] ступень нильпотентности группы $T_{n}$ не меньше $n+1$, что противоречит лемме 3.1.5 из [4].

Итак, в дальнейшем мы можем считать, что если $p$ и $q-$ различные простые числа и группа $Z_{q}$ принадлежит формации $m\left(Z_{p}\right)$, то $q \notin \pi(\mathfrak{H})$. Кроме того, как и при доказательстве теоремы 8.16 в [5], можно показать, что в этой ситуации формация $\mathfrak{H}$ абелева.

Покажем теперь, что $\mathfrak{M}-$ метанильпотентная однопорожденная $c$-формация. Предположим, что $\mathfrak{M} \nsubseteq \mathfrak{N}^{2}$. Тогда, по лемме 6 в $\mathfrak{M}$ имеется неметанильпотентная $c$-подформация $\mathfrak{M}_{0}$, у которой все собственные $c$-подформации метанильпотентны. Причем по лемме $7 \mathfrak{M}_{0}=\operatorname{cform} G$, где группа $G$ удовлетворяет одному из следующих условий:

(a) $P=G^{\mathfrak{N}^{2}}-$ неабелева единственная минимальная нормальная подгруппа в $G$,

(b) $G=[P] H$, где $P=C_{G}(P)-$ минимальная нормальная подгруппа в $G$, a $H=$ $[Q] N \neq 1, Q=C_{H}(Q)=H^{\mathfrak{N}}-$ минимальная нормальная подгруппа в $H$.

Пусть $G$ удовлетворяет условию (а) и $B \in K(P)$. Ясно, что $G \in m(B)$. Пусть $M$ - неединичная группа из $\mathfrak{H}$. Для всякого натурального числа $n$ через $G_{n}$ обозначим регулярное сплетение

$$
G_{n}=G \curlywedge M^{n}=[K] M^{n}
$$

где $K$ - база сплетения. Так как $G_{n} / K \cong M^{n} \in \mathfrak{H}$, то $\left(G_{n}\right)^{\mathfrak{H}} \subseteq K \in m(B)$. Если $G=P$, то $\left(G_{n}\right)^{\mathfrak{H}} \in m(B)$, то есть $G_{n} \in m(B) \mathfrak{H}$. Поскольку $O_{T}\left(G_{n}\right)=1$ для любой простой группы $T \in \mathfrak{I} \backslash(B)$, по теореме из [9]

$$
G_{n} \in h(B)=\text { form }\left(A / F_{B}(A)\right)
$$

для любого $n \in \mathrm{N}$. Но $G_{n}$ содержит монолит порядка $|G|^{|M|^{n}}$, что противоречит лемме 3.1.5 из [4].

Предположим, что $P \subset G$. Докажем, что в этом случае $\left(G_{n}\right)^{\mathfrak{H}}$ входит подпрямо в $K$. Пусть $D_{1}$ - проекция $\left(G_{n}\right)^{\mathfrak{H}}$ на первую копию $G_{1}$. Допустим, что $D_{1} \neq G_{1}$. Поскольку $\left(G_{n}\right)^{\mathfrak{H}}$ нормальна в $G_{n}$, то $D_{1}$ нормальна в $G_{1}$. По лемме 3.1 .9 из [4] 
$\left(G_{1} / D_{1}\right)$ 乙 $M^{n}$ - гомоморфный образ группы $G_{n} /\left(G_{n}\right)^{\mathfrak{H}}$, то есть $\left(G_{1} / D_{1}\right)$ 乙 $M^{n} \in \mathfrak{H}$. Следовательно, $\pi\left(G_{1}\right) \cap \pi(\mathfrak{H}) \neq \varnothing$. Без ограничения общности в качестве $M$ можно взять прямое произведение $q d$-групп из $\mathfrak{H}$, где $q \in \pi\left(G_{1}\right) \cap \pi(\mathfrak{H})$, по одной для каждого $q$.

Уточним строение $\mathfrak{H}$. Введем обозначение

$$
\mathfrak{F}_{1}=\operatorname{form}\left\{A / F_{B}(A) \mid B \in K(A)\right\} .
$$

Хорошо известно, что формация, порожденная конечным множеством групп, однопорождена. Значит, $\mathfrak{F}_{1}=$ form $S$ для некоторой группы $S$. Пусть $A_{1}-$ произвольная монолитическая группа из $\mathfrak{H}$. Понятно, что $O_{Z_{p}}\left(A_{1}\right)=1$ для некоторого $p \in \pi(\mathfrak{M})$. Значит, $A_{1} \in \mathfrak{H} \subseteq m\left(Z_{p}\right) \mathfrak{H}$ и по теореме из [9]

$$
A_{1} \in h\left(Z_{p}\right)=\text { form }\left(A / F_{Z_{r}}(A)\right) \subseteq \mathfrak{F}_{1} .
$$

Следовательно, всякая монолитическая группа из $\mathfrak{H}$ входит в $\mathfrak{F}_{1}$. Таким образом, $\mathfrak{H} \subseteq \mathfrak{F}_{1}$.

Если $L$ - подгруппа порядка $q$ из $M$, а $Z_{q}$ - подгруппа порядка $q$ из $G_{1} / D_{1}$, то по лемме 18.8(a) из [12], группа $T_{n}=Z_{q}$ 乙 $L^{n}$ изоморфно вкладывается в $\left(G_{1} / D_{1}\right)$ 乙 $M^{n}$. Тогда $T_{n} \in \operatorname{sform} S$ для всех $n \in \mathrm{N}$, что противоречит лемме 3.1.5 из [4]. Таким образом, $G_{1}=D_{1}$, то есть $\left(G_{n}\right)^{\mathfrak{H}}$ входит подпрямо в $K$. Поэтому $\left(G_{n}\right)^{\mathfrak{H}} \in m(B)$ и $G_{n} \in m(B) \mathfrak{H}$. Поскольку $O_{T}\left(G_{n}\right)=1$ для любой простой группы $T \in \mathfrak{I} \backslash(B)$, по теореме работы [9]

$$
G_{n} \in h(B)=\text { form }\left(A / F_{B}(A)\right) .
$$

Но $G_{n}$ содержит монолит порядка $|P|^{|M|^{n}}$, что противоречит лемме 3.1 .5 из [4].

Рассуждая так же, как при доказательстве теоремы 8.16 в [5], можно показать, что группа $G$ не может удовлетворять и условию (b). Следовательно, $\mathfrak{M} \subseteq \mathfrak{N}^{2}$.

Покажем, что $\mathfrak{M}$ - однопорожденная $c$-формация. Поскольку всякая $c$-подформация из $\mathfrak{M}$ наследственна, то $\mathfrak{M} \subseteq \mathfrak{F}$. По лемме 1 в $\mathfrak{M}$ имеется лишь конечное множество $c$-подформаций. Пусть

$$
\mathfrak{E}=\mathfrak{M}_{0} \subset \mathfrak{M}_{1} \subset \ldots \subset \mathfrak{M}_{k}=\mathfrak{M}
$$

- такая цепь $c$-формаций, что $\mathfrak{M}_{i-1}$ - максимальная $c$-подформация в $\mathfrak{M}_{i}$, $i=1,2, \ldots, k$. Пусть

$$
H_{i} \in \mathfrak{M}_{i} \backslash \mathfrak{M}_{i-1}, \quad i=1,2, \ldots, k .
$$

Тогда

$$
\mathfrak{M}=\operatorname{cform}\left\{H_{1}, \ldots, H_{k}\right\}=\operatorname{cform} H_{1} \times \ldots \times H_{k},
$$

то есть $\mathfrak{M}$ - однопорожденная $c$-формация. Итак, $\mathfrak{M}$ удовлетворяет условию 2.

Предположим, что найдется такое простое число $q$, что $q \in \pi(\mathfrak{H}) \cap \pi(m(B))$. Так как формация $\mathfrak{M}$ метанильпотентна, $B \cong Z_{p}$ для некоторого $p \in \mathbf{P}$. Причем, по следствию 1 из [9] $m\left(Z_{p}\right) \subseteq m_{1}\left(Z_{p}\right)$, где $m_{1}$ - минимальный $c$-спутник формации $\mathfrak{N}^{2}$. По теореме 7.9 из [5] $m\left(Z_{p}\right) \subseteq \mathfrak{N}$. Более того, учитывая теорему работы [9], заключаем, что $m\left(Z_{p}\right) \subseteq \mathfrak{N}_{p^{\prime}}$. Следовательно, $q \neq p$ и в формации $m\left(Z_{p}\right)$ имеется группа порядка $q$. Значит, как показано ранее, $q \notin \pi(\mathfrak{H})$. Получаем противоречие. Таким образом, $\mathfrak{M}$ и $\mathfrak{H}$ удовлетворяют условию 3. 
Пусть $\mathfrak{M} \nsubseteq \mathfrak{N}$. Тогда найдется такое $Z_{p} \in K(\mathfrak{M})$, что $\mathfrak{E} \subset m\left(Z_{p}\right)$. Ho $m\left(Z_{p}\right) \subseteq \mathfrak{N}_{p^{\prime}}$. Поэтому в формации $m\left(Z_{p}\right)$ имеется группа простого порядка $q$, где $q \neq p$. В этом случае ввиду установленного ранее формация $\mathfrak{H}$ абелева.

Предположим теперь, что $|\pi(\mathfrak{M})|>1$. Ранее отмечалось, что $\mathfrak{H} \subseteq \mathfrak{F}_{1}$. Пусть $\mathfrak{H} \nsubseteq \mathfrak{A}$. Тогда ввиду доказанного выше $\mathfrak{M} \subseteq \mathfrak{N}$. Следовательно, $m\left(Z_{p}\right)=\mathfrak{E}$ при любом $Z_{p} \in K(\mathfrak{M})$. Таким образом,

$$
f\left(Z_{p}\right)=m\left(Z_{p}\right) \mathfrak{H}=\mathfrak{H}
$$

для всех $Z_{p} \in K(\mathfrak{M})$ и $f(B)=\mathfrak{H}$ для $B \in K(\mathfrak{H}) \backslash K(\mathfrak{M})$. Но $h \leqslant f$. Значит, $A / F_{B}(A) \in \mathfrak{H}$ при всех $B \in K(A)$, то есть $\mathfrak{F}_{1} \subseteq \mathfrak{H}$. Таким образом, $\mathfrak{H}=\mathfrak{F}_{1}-$ однопорожденная формация. Пусть $\mathfrak{H} \subseteq \mathfrak{A}$. Поскольку $\mathfrak{H} \subseteq \mathfrak{F}_{1}$, по лемме 3.1 .5 из [4] число неизоморфных монолитических групп из $\mathfrak{H}$ конечно, ибо все они являются циклическими группами порядка не больше $|A|$. Значит, и в этом случае формация $\mathfrak{H}$ однопорождена.

Теорема доказана.

Замечание 2. Аналогичные результаты независимо получены и анонсированы в [13].

Теорема 2. Произведение $\mathfrak{M H}$ неединичной с-формации $\mathfrak{M}$ такой, что $\mathfrak{N}_{\pi(\mathfrak{M})} \subseteq \mathfrak{M}$ $u$ неединичной формации $\mathfrak{H} \neq \varnothing$ является однопорожденной $c$ - формацией, если выполняются следующие условия:

(1) $K(\mathfrak{H}) \subseteq K(\mathfrak{M})$

(2) $\mathfrak{M}$ - метанильпотентная однопорожденная с-формация,

(3) $\pi(\mathfrak{H}) \cap \pi(m(B))=\varnothing$ для всех $B \in K(\mathfrak{M})$, где $m$ - минималъный $c$-спутник формации $\mathfrak{M}$,

(4) если $|\pi(\mathfrak{M})|>1$, то $\mathfrak{H}$ - однопорожденная формачия, причем $\mathfrak{M} \nsubseteq \mathfrak{N}$ влечет включение $\mathfrak{H} \subseteq \mathfrak{A}$.

Доказательство. Обозначим формацию $\mathfrak{M H}$ через $\mathfrak{F}$. Пусть $\pi(\mathfrak{M})=\{p\}$. Учитывая включение $\mathfrak{N}_{\pi(\mathfrak{M})} \subseteq \mathfrak{M}$, заключаем, что $\mathfrak{N}_{p}=\mathfrak{M}$. Из условия 1 следует, что $\mathfrak{H} \subseteq \mathfrak{N}_{p}$. Значит,

$$
\mathfrak{F}=\mathfrak{M H}=\mathfrak{M}=\mathfrak{N}_{p}
$$

Следовательно, $\mathfrak{F}=\operatorname{cform}\left(Z_{p}\right)$.

Рассмотрим случай, когда $|\pi(\mathfrak{M})|>1$. Ввиду леммы 3 формация $\mathfrak{F}$ имеет такой c-спутник $f$, что $f(A)=m(A) \mathfrak{H}$, если $A \in K(\mathfrak{M})$, и $f(A)=\varnothing$ для всех $A \in \mathfrak{I} \backslash K(\mathfrak{M})$. По условию $2 \mathfrak{M}$ - однопорожденная $c$-формация. Значит, по следствию 6 из [8] $K(X \mathfrak{M})=K(X \mathfrak{F})-$ конечное множество.

Используя рассуждения, приведенные в доказательстве теоремы 8.16 в [5], несложно показать, что формация $m(A) \mathfrak{H}$ для любого $A \in K(\mathfrak{M})$ - однопорождена. Тогда по лемме 3.5.21 из [4] $\mathfrak{F}$ является однопорожденной $c$-формацией.

Теорема доказана. 


\section{Список литературы}

1. Скиба А. Н., О произведении формаций. Алгебра и логика (1983) 22, №5, 574-583.

2. Скиба А. Н. О факторизациях одного класса формаций конечных групп. Bопросы алгебры (1992) 7, 108-110.

3. Skiba A. N., On nontrivial factorizations of an onegenerated local formation of finite groups. In: Proc. Int. Conf. Algebra Dedicat. Mem. A. I. Malcev, Novosibirsk, 21-26 Aug. 1989, p. 111.

4. Скиба А.Н., Алгебра формачий. Беларуская навука, Минск, 1997.

5. Шеметков Л. А., Скиба А. Н., Формации алгебраических систем. Наука, Москва, 1989.

6. Шеметков Л. А., Формачии конечных групп. Наука, Москва, 1978.

7. Ведерников В. А., Сорокина М. М., О композиционных наследственных критических формациях. Препринт №1., Брянск, БГПУ, 1996.

8. Еловиков А. Б., Кратно $\Omega$-композиционные формации групп. Препринт Ne3, Брянск, БГПУ, 1999.

9. Скиба А. Н., Шеметков Л. А., О минимальном композиционном экране композиционной формации. Вопросъ алгебры (1992) 7, 39-43.

10. Еловиков А. Б., О факторизации формаций. Тезисы межд. науч. конф., посвященной 80-летию проф. Волъфганга Гашюча. Гомель, 16-21 октября 2000 г., Гомельский гос. ун-т., Гомель, 23-25.

11. Сорокина М. М., О композиционных и локальных критических формациях. Изв. вузов. Сер. математиха (2000), №7, 59-66.

12. Doerk K., Hawkes T.; Finite soluble groups. Gruyter, Berlin, 1992.

13. Го Вэньбинь, Скиба А. Н., Факторизации однопорожденных композиционных формаций. В сб.: Тезисы IV Межд. алгебр. конф., посвященной 60-летия проб. Ю. И. Мерзлякова. Новосибирск, 7-11 августа 2000 г., Институт математики СО РАН, Новосибирск, с. 60-61.

Статья поступила 06.03.2001.

«ДИСКРЕТНАЯ МАТЕМАТИКА , 2001, ТОМ 13, ВЫПУСК 3

Заведующая редакцией Л. М. Барыкина

Сдано в набор 8.08.2001. Подписано к печати 28.08.2001. Формат 70 $100 / 16$.

Печать офсетная. Усл. печ.л. 13,0. Усл. кр.-отт. 3,1 тыс. Уч.-изд.л. 12,6. Бум.л. 5,0. \begin{tabular}{ll} 
Тираж 234 экз. Заказ N2448. \\
\hline
\end{tabular}

Свидетельство о регистрации №1868 от 28.01.1991 г. в Госкомпечати СМ СССР.

Учредители: Академия наук СССР, Отделение математики

Адрес издательства: 117997 г. Москва, Профсоюзная ул., д. 90.

Адрес редакции: 117966 г. Москва ГСП-1, ул. Губкина, д. 8, комн. 622. Тел. 9383700.

Отпечатано в ППП “Типография "Наука"

121099 г. Москва Г-99, Шубинский пер., д. 6.

Налоговая льгота - общероссийский классификатор продукции ОК-005-93, том 2;

952000 - журналы 